\title{
Could COVID-19 Reverse the Modest Gains Made in Newborn Health in Ethiopia?
}

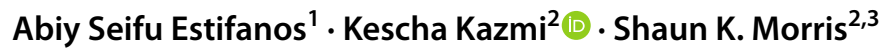

Accepted: 26 April 2021 / Published online: 3 May 2021

(c) Crown 2021

\begin{abstract}
Introduction Ethiopia has made remarkable progress in reducing childhood and neonatal mortality in the last two decades. However, with the spread of the COVID-19 pandemic in Ethiopia, disruptions in routine health care pose a significant risk in reversing the gains made in neonatal mortality reduction.

Methods Using the World Health Organization's health systems building blocks framework we examined the mechanisms by which the pandemic may impact neonatal health.

Results Our analysis suggests that the COVID-19 pandemic and measures taken by the government to control its spread could indirectly set back the gains made in neonatal mortality reduction in Ethiopia by weakening the health system building blocks. On the other hand, by exposing longstanding issues in the health system, the pandemic has pressed health sector stakeholders to urgently test innovative approaches to maintain delivery of essential health care.

Conclusions We recommend that the Ministry of Health of Ethiopia strike a right balance between the control of the pandemic and ensuring provision of essential neonatal health services. As the pandemic continues to spread in the country, the government should avoid verticalization of pandemic response efforts and adopt a diagonal investment approach to effectively respond to the pandemic as well as build health system resilience to maintain the gains made in the neonatal health.
\end{abstract}

Keywords COVID-19 Coronavirus $\cdot$ Newborn health $\cdot$ Maternal health

\section{Significance}

The 2014-2016 Ebola epidemic in West Africa led to significant disruptions in maternal and newborn health services and subsequent poor outcomes. Modeled estimates developed during the earlier phase of the COVID-19 pandemic project excess maternal and child mortality due to the disruption of maternal and child health services. Our paper adds to this knowledge by examining the mechanism by which the COVID-19 pandemic may impact neonatal health

Kescha Kazmi

Kescha.kazmi@sickkids.ca

1 Department of Reproductive, Family and Population Health, School of Public Health, Addis Ababa University, Addis Ababa, Ethiopia

2 Division of Infectious Diseases, Department of Pediatrics, Hospital for Sick Children, 555 University Ave, Toronto, ON M5G 1X8, Canada

3 Centre for Global Child Health, Child Health Evaluative Sciences, Hospital for Sick Children, Toronto, ON, Canada care services in Ethiopia and set back the gains made in the last two decades using the World Health Organization's health system building blocks framework as a lens.

\section{Introduction}

Ethiopia is one of the few countries in the world that achieved the United Nations Millennium Development Goal Four (MDG4) of reducing child mortality by two thirds three years ahead of the target year of 2015. The under-five morality rate was reduced from 205 deaths per 1000 live births in 1990 to 68 deaths per 1000 live births in 2012. During the same time frame, the neonatal mortality rate went from 59 to 34 deaths per 1000 live births (United Nations Interagency Group for Child Mortality Estimation, 2018). Building on this success, the country aimed to further decrease the under-five, infant and neonatal mortality rates to less than 30, 20, and 10 per 1000 live births respectively by 2020 (Federal Ministry of Health of Ethiopia, 2015). 
However, on March 11, 2020 the World Health Organization (WHO) declared Coronavirus disease 2019 (COVID19) a global pandemic. As of February 9, 2021, a total of 142,338 cases and 2,148 deaths have been confirmed in Ethiopia, which is likely to be an underestimate of the true number of cases due to both testing policy and limitations in test availability (World Health Organization, 2021). If routine health care, essential for maintaining and continuing reduction in mortality gains, is disrupted due to COVID-19 in Ethiopia, the increase in newborn mortality as an indirect effect of the pandemic may be substantial (Roberton et al., 2020). In this narrative review we assess the potential indirect effects of COVID-19 on the gains made in neonatal health in Ethiopia.

\section{How Would COVID-19 Affect Newborn Health in Ethiopia?}

The WHO's health systems building blocks framework consists of 6 building blocks, health service delivery, health workforce, health information systems, access to essential medicines, financing, and leadership (World Health Organization, 2010). We examined the potential effects of COVID19 by applying the WHO's health systems building blocks framework as a lens to guide the discussion on how the pandemic may setback the gains made in newborn health in Ethiopia (Fig. 1).

\section{Health Service Delivery}

During the 2014-2016 Ebola epidemic in West Africa, the general public had significant distrust of Ebola treatment centers as the medical system at the time actually amplified virus transmission. More than 300 doctors and nurses died due to lack of implementing appropriate outbreak control and containment measures (Elargoubi et al., 2017). As a result, a significant reduction in antenatal care utilization and births attended at a healthcare facility were seen during the epidemic. For women that did access care, there was a corresponding increase in facility maternal mortality ratio and stillbirth rate (Jones et al., 2016). Similarly, the delivery of safe and effective neonatal health services to all newborns and caretakers could be severely impacted by the COVID-19 pandemic in Ethiopia in a number of ways.

First, the uptake of neonatal health care services could decrease. In the earlier phase of the pandemic in Ethiopia, the care seeking for maternal and neonatal health services declined, blood donations shrank, essential surgical care such as caesarean section became unpredictable, transportation of essential drugs and medical supplies was disrupted, and some facilities entirely closed their gates and turned back people who would come to receive services (Marks, 2020; Mohammadi et al., 2020). Other medical services providing tuberculous and HIV care similarly saw a dramatic decline in service utilization (Melaku \& Assefa, 2020; Mohammed et al., 2020). In Dire Dawa, TB cases detected were three times lower in the spring of 2020 compared to previous reporting periods (Mohammed et al., 2020).
Fig. 1 Mechanisms by which the COVID-19 pandemic could influence neonatal health in Ethiopia using the WHO's health system building blocks framework. COVID 19 coronavirus disease 2019, GDP gross domestic product, HMIS health management information systems, $M N H$ maternal newborn health, $H E W s$ health extension workers, EmONC emergency obstetric and newborn care, $C / S$ caesarian section, IPAC infection prevention and control

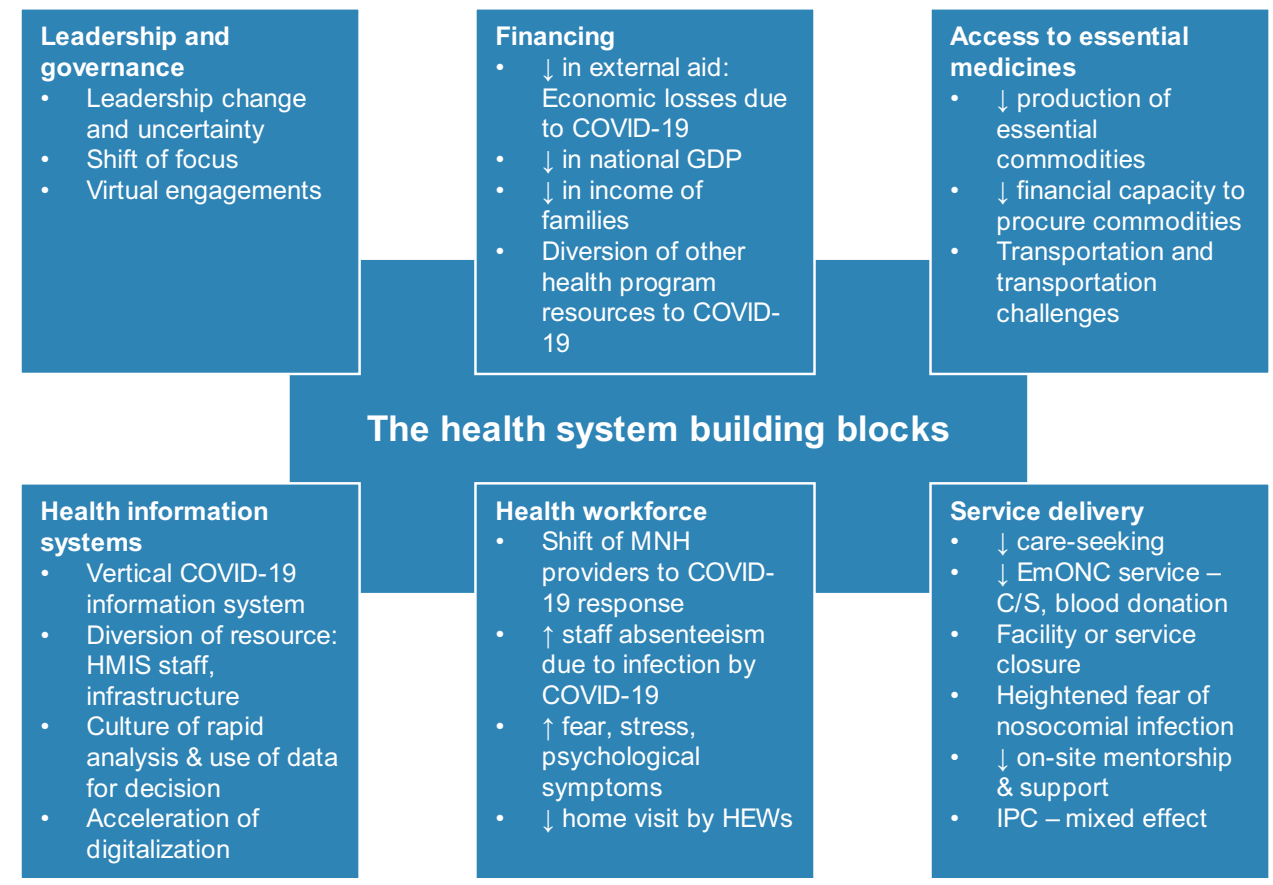


Political decisions to impose an early State of Emergency at the national level on April 8, 2020 (The Federal Democratic Republic of Ethiopia House of Representatives, 2020) might have contributed to significant fear of COVID-19 among health care providers and communities alike. Other reasons include lack of education on COVID-19 (Kebede et al., 2020) and inability of the ministry of health and regional health bureaus to provide clear guidance and direction to health facilities and communities as they were figuring out the nature and scale of the pandemic and how to respond to it. To counter the sudden decline of health care seeking, the ministry of health later advised communities to continue using maternal and neonatal health services and health facilities to continue providing essential medical services. As seen during the 2014-2016 West Africa Ebola outbreak, rebuilding trust of the community towards the healthcare system and regaining pre-pandemic health care utilization coverage after it drops during an outbreak can be challenging.

Second, neonatal health care safety could be compromised. The decline of uptake of maternal and neonatal health services is partly due to fear of safety in healthcare settings among health workers and the caretakers. In the absence of clearly outlined strategies on health care safety and in the face of scarce resources, guidelines, tools, and supplies, ensuring safety remains unclear. COVID-19 has further exposed these long-standing health system challenges. Public health measures to control the spread of COVID-19 implemented in high-income countries, such as physical distancing and use Personal Protective Equipment (PPE) has further strained the provision of safe maternal and neonatal healthcare in Ethiopia (Freckelton, 2020). The lack of easily accessible maternal and neonatal health services could force families to seek care from less safe traditional practitioners.

Third, the COVID-19 pandemic poses a high risk of losing the gains made in designing, introducing, and institutionalizing quality improvement interventions in maternal and neonatal health services. The ministry of health recognized the need for provision of high quality maternal and neonatal health services both to improve the health outcomes and enhance the experiences of clients and health care providers. Consequently, in addition to developing a national quality strategy, the ministry made efforts to test and implement different maternal and newborn health quality improvement interventions across the three tiers of the health system (Magge et al., 2019). National scale-up of these interventions requires strong leadership commitment and resources to build capacity across all levels and create learning systems that facilitate robust quality planning, control and improvement (Federal Democratic Republic of Ethiopia Ministry of Health, 2016). During the pandemic, focus shifted from routine essential care to the prevention and control of COVID-19 pandemic. This could undermine the pace and scale of implementation of maternal and newborn health quality improvement interventions.

Fourth, the COVID-19 pandemic could affect the neonatal healthcare infection prevention and control (IPAC) practices both positively and negatively. Before this pandemic, IPAC practices in health facilities in Ethiopia were generally suboptimal (Desta et al., 2018). The COVID-19 global, national and local infection prevention campaigns, particularly handwashing and wearing masks are believed to improve the public awareness and practices of infection prevention and control (Yousuf et al., 2020). The Ethiopian government lifted the excise tax on denatured alcohol and as a result, many hospitals started to prepare and distribute hand-sanitizers to their staff, other health facilities, and communities (Gelila, 2020). Several small start-up businesses and existing factories have also joined the hand-sanitizer and mask production and distribution (Sahlu, 2020). There is a need to empirically measure the effect of these IPAC measures and campaigns on neonatal nosocomial infection, morbidity and mortality. Conversely, introducing excessive infection prevention precautions in settings where there are no adequate supplies may incapacitate the maternal and neonatal health care providers from providing essential lifesaving care. Furthermore, maternal and neonatal health IPAC resources could be diverted to COVID-19 prevention and control, which could worsen the infection prevention practices at maternity and neonatal care wards (Stevens et al., 2020).

Fifth, the COVID-19 pandemic pressed Ethiopia to seek local innovations to fight the pandemic. With all its challenges, the COVID-19 pandemic pushed many institutions in Ethiopia to become increasingly innovative. The ministry of health and scores of hospitals in Ethiopia have been working with engineers, social-scientists and industries to repair ventilators, produce PPE and develop non-pharmaceutical solutions to mitigate the impact of COVID-19 pandemic. This experience enabled different stakeholders within the country to appreciate the needs and capacities across sectors and both build on existing and forge new partnerships that may go beyond the pandemic. With the pandemic-related decreases in field activities and supervision, health development partners are now considering use of remote training and support technologies. With no defined end date for COVID-19, harnessing the potentials of E-health and digital health services in the immediate future is likely (Chersich et al., 2020).

\section{Health Workforce}

Provision of standard neonatal health services requires a sufficient number of skilled health workers. The health workforce in Ethiopia has improved following a massive scale 
up of training and education by the government (Federal Ministry of Health of Ethiopia, 2015). Although maternal newborn health $(\mathrm{MNH})$ care has benefited from this investment, there is still a shortage of key $\mathrm{MNH}$ professionals (Haileamlak, 2018). This shortage is made worse by high turnover of the MNH staff, limited competency and motivation, and challenges related to training, deployment, and retention (Assefa et al., 2017).

The COVID-19 pandemic threatens to further reduce the availability of competent and motivated maternal and neonatal healthcare providers. As seen in other countries, the COVID-19 pandemic diverts health workers towards COVID-19 efforts (Carenzo et al., 2020). Critical healthcare worker shortages are intensified by healthcare worker absenteeism due sicknesses, underlying health conditions that put them at higher risk, and psychological stress from COVID-19 (Spoorthy et al., 2020). For health extension workers in Ethiopia, shortage of PPE and the need to keep physical distancing to prevent transmission of COVID-19 complicates home visits and care provision.

\section{Health Information System}

In the last 5 years, Ethiopia was striving to revolutionize its existing health management information systems (HMIS) through transforming the collection, aggregation, analysis and use of data at all levels using digital, automation and information and communications technologies (ICT) (Federal Ministry of Health of Ethiopia, 2015). The COVID-19 pandemic pressed the country to trial some of these novel technologies on a larger scale. Within a span of three months, numerous digital and online platforms have been adapted and/or developed to facilitate recording, analysis, reporting and use of COVID-19 data ("Nation Launches Mobile Applications to Control COVID-19," 2020). Through the COVID-19 response, the Ministry of Health, Ethiopian Public Health Institute and other health development partners were able to appreciate the power of generating, analyzing and using data to inform decisions. If institutionalized and normalized within the HMIS, these novel ICT technologies could be utilized within neonatal care.

However, the verticalization of the COVID-19 information system could further weaken the poorly organized HMIS by diverting health information technicians and ICT infrastructure towards the COVID-19 response. Essential staff involved in recording, aggregating, and analyzing the routine HMIS data could be absent from work due to sickness or fear of COVID-19. In both instances, the recording, analysis, reporting and use of neonatal health care data might be affected.

\section{Essential Medical Products, Vaccines and Technologies}

Safe, efficacious and cost-effective medical products, vaccines and technologies are essential to provision of both preventative and curative neonatal health services. Globally, the production of medical products, vaccines and technologies have been slowed due to the COVID-19 pandemic. Lowto-middle income countries' capacity to procure essential neonatal health commodities are compromised by economic losses from COVID-19 pandemic, countries restricting export of medical supplies, as well as the shrinking global Development Assistance for Health (DAH). Furthermore, transportation of essential commodities through global supply chains becomes a challenge due to the travel bans and restrictions imposed across borders. Ethiopia already faced a chronic shortage of essential neonatal health drugs and commodities (Services Availability and Readiness Assessment (SARA) 2018 Final Report, 2018). The COVID-19 pandemic could worsen the shortage by disrupting procurement and importation, and transportation of essential neonatal health commodities.

\section{Health Care Financing}

The health systems building block that is most likely to be affected by the COVID-19 pandemic is health care financing. The DAH has stagnated long before the COVID-19 pandemic, forcing low-income countries to look for domestic financing options (Chang et al., 2019). The pandemic has led to trillions of dollars in economic losses globally, which will eventually have a trickledown effect on the national economies of low-income countries like Ethiopia. Ethiopia's health care financing largely depends on external aid and out-of-pocket expenditures by users (World Health Organization, 2020). Countries that typically provide external aid have shifted attention to their own country's response to the COVID-19 pandemic. The shrinking global and national economy will have detrimental consequences on neonatal health in at least two ways.

First, the neonatal health program is heavily reliant on external aid, thus vulnerable to disruptions to the global and national economies. Unless meaningful financial prioritization at global and national levels is done to channel sufficient funds for the maternal and neonatal health programs, the modest gains made in neonatal health could be lost. Families may not have access to quality essential neonatal health care without experiencing financial catastrophe. Without adequate funding, all other health systems building blocks cannot be strengthened, challenging the country's goal for universal neonatal health coverage. 
Second, efforts to contain the spread of COVID-19 will compete for resources with other existing routine health programs (Emanuel et al., 2020). The absence of substantial external support combined with the internal economic slowdown and political sensitivity of the pandemic will likely divert resources from the existing health programs to the COVID-19 pandemic. The effect of this shift of finance on the provision of neonatal health will become considerable as COVID-19 spreads throughout the country.

\section{Leadership and Governance}

Strong leadership commitment is fundamental to implement universal coverage of health care services including neonatal health care (Admasu, 2016). The health system leadership is situated within the bigger political environment. In the last two years, the Ethiopian government has been in a state of political transition. Following the leadership change within the ruling party in April 2018, there was change of the minister of health as the new prime minister formed his cabinet. Ever since, there were three more rounds of reshuffling, one of which resulting in the replacement of the minister of health after about a year and half in office. In April 2020, the National Electoral Board decided to postpone the national election due to the COVID-19 pandemic. This decision created a heightened tension between the ruling and opposition parties as the latter pushed the government to share power. Politically motivated conflicts and social unrests observed in different parts of the country coupled with a major locust infestation have only intensified the climate of uncertainty in Ethiopia (Anna, 2020). The ruling party may find itself in another round of cabinet reshuffling to appease different interest groups in the near future, which may involve replacing the health minister for the third time.

Although Ethiopia's health programs and priorities are often minimally affected by change of the political leadership, the transitional nature of the leaders during the COVID-19 pandemic may shift their focus away from strategic systemwide priorities to popular issues. The middle and lower level leaders often follow suit creating vacancy in leadership support that the health system needs to realize universal health coverage, including provision of high-quality neonatal health. The experience since the first COVID-19 case was reported in Ethiopia demonstrates how the priorities of the health system leaders can easily shift. Consequently, the country may be challenged both to maintain the moderate gains made in neonatal health as well as to accelerate progress to meet the national and United Nation's Sustainable Development Goals (SDG) targets.

\section{Conclusions and Recommendations}

In this narrative review we assessed the indirect effects of COVID-19 on the moderate gains made in neonatal health in Ethiopia. Our analysis suggests that COVID-19 pandemic could easily reverse the modest gains made in neonatal health through weakening the building blocks of the health system. Ethiopia needs to strike the right balance between taking drastic measures to control the COVID-19 pandemic and losing the gains made in existing neonatal health programs. As the country experiences increasing numbers of COVID-19 cases and enhances its response, it should move away from verticalization of the response efforts and adopt a diagonal investment approach to build health system resilience and maintain the gains made in neonatal health.

\section{Declarations}

Conflict of interest Authors declare they have no conflict of interest.

\section{References}

Admasu, K.-B. (2016). Designing a resilient national health system in Ethiopia: The role of leadership. Health Systems and Reform, 2(3), 182-186. https://doi.org/10.1080/23288604.2016.1217966

Anna, C. (2020, November 19). Ethiopia's multiple crises: War, COVID-19, even locusts. AP News. https://apnews.com/article/ ethiopia-faces-war-virus-locusts-604afde8881b253de700c8ec3 $60 \mathrm{f} 7673$

Assefa, T., Haile Mariam, D., Mekonnen, W., \& Derbew, M. (2017). Health system's response for physician workforce shortages and the upcoming crisis in Ethiopia: A grounded theory research. Human Resources for Health, 15(1), 1-11. https://doi.org/10. 1186/s12960-017-0257-5

Carenzo, L., Costantini, E., Greco, M., Barra, F. L., Rendiniello, V., Mainetti, M., Bui, R., Zanella, A., Grasselli, G., Lagioia, M., Protti, A., \& Cecconi, M. (2020). Hospital surge capacity in a tertiary emergency referral centre during the COVID-19 outbreak in Italy. Anaesthesia, 75(7), 928-934. https://doi.org/10.1111/ anae. 15072

Chang, A. Y., Cowling, K., Micah, A. E., Chapin, A., Chen, C. S., Ikilezi, G., Sadat, N., Tsakalos, G., Wu, J., Younker, T., Zhao, Y., Zlavog, B. S., Abbafati, C., Ahmed, A. E., Alam, K., Alipour, V., Aljunid, S. M., Almalki, M. J., Alvis-Guzman, N., ... Dieleman, J. L. (2019). Past, present, and future of global health financing: A review of development assistance, government, out-of-pocket, and other private spending on health for 195 countries, 1995-2050. The Lancet, 393(10187), 2233-2260. https://doi.org/10.1016/ S0140-6736(19)30841-4

Chersich, M. F., Gray, G., Fairlie, L., Eichbaum, Q., Mayhew, S., Allwood, B., English, R., Scorgie, F., Luchters, S., Simpson, G., Haghighi, M. M., Pham, M. D., \& Rees, H. (2020). COVID-19 in Africa: Care and protection for frontline healthcare workers. Globalization and Health, 16(1), 46. https://doi.org/10.1186/ s12992-020-00574-3

Desta, M., Ayenew, T., Sitotaw, N., Tegegne, N., Dires, M., \& Getie, M. (2018). Knowledge, practice and associated factors of infection 
prevention among healthcare workers in Debre Markos referral hospital Northwest Ethiopia. BMC Health Services Research, 18(1), 1-10. https://doi.org/10.1186/s12913-018-3277-5

Elargoubi, A., Khelifa, A. W. B., Haddad, O., Mhalla, S., Sakly, N., \& Mastouri, M. (2017). Lessons about causes and management of an ebola outbreak. Folia Medica, 59(4), 387-395. https://doi.org/ 10.1515/folmed-2017-0032

Emanuel, E. J., Persad, G., Upshur, R., Thome, B., Parker, M., Glickman, A., Zhang, C., Boyle, C., Smith, M., \& Phillips, J. P. (2020). Fair allocation of scarce medical resources in the time of covid-19. New England Journal of Medicine, 382(21), 2049-2055. https:// doi.org/10.1056/NEJMsb2005114

Federal Democratic Republic of Ethiopia Ministry of Health. (2016). Ethiopian National Health Care Quality Strategy 2016-2020. Federal Democratic Republic of Ethiopia Ministry of Health.

Federal Ministry of Health of Ethiopia. (2015). Health Sector Transformation Plan 2015/16-2019/20 (2008 - 2012 EFY).

Freckelton, I. (2020). COVID-19, negligence and occupational health and safety: Ethical and legal issues for hospitals and health centres. Journal of Law and Medicine, 27(3), 590-600

Gelila, S. (2020, March 21). Ministry waives excise tax on denatured alcohol. Addis Fortune News. https://addisfortune.news/ministrywaives-excise-tax-on-denatured-alcohol/

Haileamlak, A. (2018). How can Ethiopia mitigate the health workforce gap to meet universal health coverage? Ethiopian Journal of Health Sciences, 28(3), 249-250. https://doi.org/10.4314/ejhs. v28i3.1

Jones, S. A., Gopalakrishnan, S., Ameh, C. A., White, S., \& Van Den Broek, N. R. (2016). 'Women and babies are dying but not of Ebola': The effect of the Ebola virus epidemic on the availability uptake and outcomes of maternal and newborn health services in Sierra Leone. BMJ Global Health. https://doi.org/10.1136/ bmjgh-2016-000065

Kebede, Y., Yitayih, Y., Birhanu, Z., Mekonen, S., \& Ambelu, A. (2020). Knowledge, perceptions and preventive practices towards COVID-19 early in the outbreak among Jimma university medical center visitors Southwest Ethiopia. Plos ONE, 15(5), 1-15. https:// doi.org/10.1371/journal.pone.0233744

Magge, H., Kiflie, A., Nimako, K., Brooks, K., Sodzi-Tettey, S., Mobisson-Etuk, N., Mulissa, Z., Bitewulign, B., Abate, M., Biadgo, A., Alemu, H., Seman, Y., Kassa, M., Barker, P., \& Burrsa, D. G. (2019). The Ethiopia healthcare quality initiative: Design and initial lessons learned. International Journal for Quality in Health Care, 31(10), G180-G186. https://doi.org/10.1093/intqhe/mzz127

Marks, S. (2020, April 10). COVID-19 limits force Ethiopian mothers to give birth at home. Voice of America News. https://www.voane ws.com/science-health/coronavirus-outbreak/covid-19-limitsforce-ethiopian-mothers-give-birth-home

Melaku, T., \& Assefa, D. (2020). COVID-19 pandemic as "Curate's Egg" on human immunodeficiency virus infection: A commentary. HIV/AIDS-Research and Palliative Care, 12, 735-737. https://doi.org/10.2147/HIV.S282374

Mohammadi, S., TabatabaeiYazdi, S. M., Eshghi, P., \& Norooznezhad, A. H. (2020). Coronavirus disease 2019 (COVID-19) and decrease in blood donation: Experience of Iranian Blood Transfusion Organization (IBTO). Vox Sanguinis, 2019, 1-2. https://doi.org/ 10.1111/vox. 12930

Mohammed, H., Oljira, L., Roba, K. T., Yimer, G., Fekadu, A., \& Manyazewal, T. (2020). Containment of COVID-19 in
Ethiopia and implications for tuberculosis care and research. Infectious Diseases of Poverty, 9(1), 1-8. https://doi.org/10.1186/ s40249-020-00753-9

Nation Launches Mobile Applications to Control COVID-19. (2020, May 30). Ethiopian News Agency. https://www.ena.et/en/?p= 14868

Roberton, T., Carter, E. D., Chou, V. B., Stegmuller, A. R., Jackson, B. D., Tam, Y., Sawadogo-Lewis, T., \& Walker, N. (2020). Early estimates of the indirect effects of the COVID-19 pandemic on maternal and child mortality in low-income and middle-income countries: A modelling study. The Lancet Global Health, 8(7), e901-e908. https://doi.org/10.1016/S2214-109X(20)30229-1

Sahlu, S. (2020, March 30). Close to 40 Firms Start Sanitizer Production. Ethiopian Monitor. https://ethiopianmonitor.com/2020/03/ 30/close-to-40-firms-start-sanitizer-production/

Services Availability and Readiness Assessment (SARA) 2018 Final Report. (2018). https://www.ephi.gov.et/images/pictures/downl oad_2011/Ethiopia-Service-Availability-and-Rediness-Asses sment-SARA-report-2018.pdf

Spoorthy, M. S., Pratapa, S. K., \& Mahant, S. (2020). Mental health problems faced by healthcare workers due to the COVID-19 pandemic-A review. Asian Journal of Psychiatry, 51(January), 102119. https://doi.org/10.1016/j.ajp.2020.102119

Stevens, M. P., Doll, M., Pryor, R., Godbout, E., Cooper, K., \& Bearman, G. (2020). Impact of COVID-19 on traditional healthcare associated infection prevention efforts. Infection Control and Hospital Epidemiology. https://doi.org/10.1017/ice.2020.141

The Federal Democratic Republic of Ethiopia House of Representatives. (2020). The House of People's Representatives has reviewed and approved the draft state of emergency issued by the Council of Ministers. https://www.hopr.gov.et/web/guest/-/the-house-ofpeople-s-representatives-has-reviewed-and-approved-the-draftstate-of-emergency-issued-by-the-council-of-ministers-

United Nations Inter-agency Group for Child Mortality Estimation. (2018). Child Mortality Estimates. https://childmortality.org/

World Health Organization. (2010). Monitoring the building blocks of health systems: A handbook of indicators and their measurement strategies. https://www.who.int/healthinfo/systems/WHO_ MBHSS_2010_full_web.pdf?ua=1

World Health Organization. (n.d.). Global Health Expeditures Database. Retrieved July 30, 2020, from https://apps.who.int/nha/datab ase

World Health Organization. (2021). WHO Coronavirus Disease (COVID-19) Dashboard. https://covid19.who.int/

Yousuf, H., Corbin, J., Sweep, G., Hofstra, M., Scherder, E., van Gorp, E., Zwetsloot, P. P., Zhao, J., van Rossum, B., Jiang, T., Lindemans, J.-W., Narula, J., \& Hofstra, L. (2020). Association of a public health campaign about coronavirus disease 2019 promoted by news media and a social influencer with self-reported personal hygiene and physical distancing in the Netherlands. JAMA Network Open, 3(7), e2014323. https://doi.org/10.1001/jamanetwor kopen.2020.14323

Publisher's Note Springer Nature remains neutral with regard to jurisdictional claims in published maps and institutional affiliations. 